Scientiæ studia, São Paulo, v. 4, n. 3, p. 473-84, 2006

\title{
त्री \\ El contexto de implicación: capacidad tecnológica y valores sociales
}

\author{
Fernando Tula Molina
}

\begin{abstract}
电
RESUMEN

El trabajo presenta un nuevo contexto socio-epistémico para la discusión de los problemas que afectan a la relación ciencia-sociedad. Bajo la idea de implicación y el análisis de los diversos modos en que la ciencia impacta en la sociedad, se proponen distinciones conceptuales para contribuir a una reflexión equilibrada, o que al menos tiene como objetivo facilitar el arribar al consenso entre actores, fines y valores diferentes. Se destaca la importancia de los entornos institucionales de reflexión y decisión sobre la ciencia frente a la dimensión pragmática de los problemas. Se destaca también la naturaleza tanto teórica como social de los problemas afectados por tales decisiones y la necesidad de abordar su discusión de modo simultáneo (y no de modo parcelado como proponen los contextos epistemológicos habituales).
\end{abstract}

PALABRAS-CLAVE $•$ Filosofía de la tecnología. Contexto de implicación. Epistemología.

Política científica.

\section{TeGnOlogía Y SOGIEDAD}

Para dimensionar el impacto de la ciencia en la sociedad es importante tener en cuenta su carácter doble. Por un lado, impacta de modo directo a través del modo en que las diversas aplicaciones tecnológicas modifican las prácticas y las relaciones concretas entre los individuos. Por el otro, de un modo indirecto pero mucho más profundo, impacta de modo simbólico por el propio valor de la eficacia como valor social, mediante la asociación ciencia-eficacia.

Esta asociación ha venido de fuentes diversas, pero desde el positivismo hasta la nanotecnología, en general bajo el supuesto de que la ciencia, y su desarrollo, redundará en beneficio de la sociedad, y su desarrollo. También la propia reflexión académica sobre la ciencia supuso inicialmente la posibilidad de garantizar el carácter universal del conocimiento científico y, de este modo, la posibilidad de un discurso unificador, superador de las diferencias entre los hombres, y aplicable para todos por igual. La heurística aplicada a tratar de comprender el curso de la naturaleza dio a luz 
teorías y prácticas que posibilitaron pensar en diversas maneras de acelerar o cambiar tal curso natural.

En muchas ocasiones no puede dejar de valorarse positivamente el disponer de mecanismos que aceleren nuestra velocidad de respuesta, o la eficacia con la que nos permite satisfacer nuestras necesidades y deseos. Queda por lamentar, sin embargo, los casos en que los propios deseos son creados o quedan fijados por tales mecanismos (convirtiéndolos en definitiva en fines), o aquellos en que la velocidad con que debemos responder olvida el tiempo necesario para apropiarnos de una determinada respuesta como nuestra. De modo general, debemos lamentar el que, en contra de los fines supuestos, la distribución de los beneficios de las prácticas científicas no se de en un marco de equidad y justicia. En tales casos, es lícito preguntarnos sobre la legitimidad del discurso tecnológico, de sus prácticas, de los fines que persigue y de sus consecuencias.

En mi opinión, la reflexión simultánea sobre lo eficaz y lo legítimo se ha tornado urgente. El valor simbólico de la eficacia parece haber prejudicado el reconocimiento de que la discusión central es sobre fines, sobre valores proyectados a futuro, sobre las discrepancias y los caminos hacia el consenso.

\section{LO HUMANO Y LO TEGNOLÓGIGO: LA POSIBILIDAD DE ELEGGIÓN}

Creo que el problema central de esta reflexión ha sido bien descripto por Robert S. Cohen al decir que:

Debemos preguntarnos si la tecnología puede tener una escala humana, si las amenazas globales ineludiblemente requieren tecnología de elite, si los beneficios a corto y mediano plazo no acarrean penas mayores en un plazo mayor, si los milagros de la ciencia reemplazan a los milagros de la religión, y la retórica de la tecnología desplaza persuasivamente a la retórica de la religión; de hecho, preguntarnos si la cohesión social puede ser preservada [...].

Estamos lejos de una comprensión satisfactoria de las alegrías y tristezas, los logros y las frustraciones de la saga tecnológica de las sociedades modernas. Debe haber todavía alternativas entre las tecnologías y debemos trabajar como científicos, tecnólogos y filósofos y prever los peligros y las oportunidades, para elegir con el sentido de todavía genuina posibilidad de cumplir valores humanos (1983, p. 47 ).

Ahora bien, la posibilidad de elegir es una posibilidad propia del hombre. Sin embargo, para la mayoría, pensar en cambiar el curso del desarrollo científico-tecno- 
lógico está casi tan alejado como pensar en cambiar el curso de la naturaleza. La posibilidad de que la ciencia y la tecnología cumplan valores humanos depende de las decisiones tomadas en los cuerpos colegiados, académicos y políticos, donde se dan las condiciones para el debate, y el entorno de legitimidad para los acuerdos. Tales decisiones son de naturaleza teórica y social, ya que se relacionan con cuáles investigaciones e implementaciones incentivar y/o regular. En tales decisiones está condensada la dimensión pragmática del problema bajo un triple eje, ético, político y epistemológico.

De antemano es difícil garantizar la decisión correcta, porque la corrección implica siempre una valoración no siempre hegemónica y puede estar sujeta a objetivos diferentes. Sin embargo, podemos decir que, por su propia naturaleza, tales decisiones en principio mejoran cuando se dispone de mayor información, cuando se logra una mayor comprensión, y cuando emanan de un mayor acuerdo. El problema general consiste en las siguientes dos preguntas: ¿cómo pueden adjudicarse las afirmaciones de los individuos, las corporaciones, el estado y otros grupos involucrados? ¿Cómo encontrar un equilibrio entre las posibilidades, los intereses, los valores, las necesidades y las responsabilidades?

En muchos casos la mera posibilidad de mejora, evaluada sobre un sólo parámetro (por ejemplo, mayor velocidad, mayor resistencia, mayor alcance, mayor definición, productividad etc.), parece ser suficiente para legitimar la inversión de los recursos necesarios para concretar tales posibilidades. Si, además, hay quienes ven la posibilidad de capitalizar los beneficios realizando las inversiones correspondientes, parecen estar dados todos los factores para concretar la mejora, y pensar en términos de progreso.

Mucho se ha dicho, sin embargo, sobre la falacia de pensar que lo novedoso implique progreso; baste notar aquí que la discusión sobre valores, necesidades concretas y responsabilidades posteriores no es imprescindible para concretar las posibilidades tecnológicas. Dicho de otro modo, la maquinaria tecnológica y la concreción de las posibilidades materiales de transformación son independientes de la discusión que pauta los fines y los modos de tal transformación, quedando tales fines muchas veces ligados sólo a los intereses de cada sector involucrado.

Al no ser necesaria, parte de la dificultad de la discusión sobre fines consiste en que hay que articularla desde afuera, buscando encausar un proceso ingenieril y tecnológico que dispone de una dinámica propia.

Quiero comenzar aquí a referirme, como también lo haré más adelante, a la obra de Hugh Lacey, cuya perspectiva general implica:

disminución del poder, no hacer todo lo que la técnica nos posibilita y mantener las cuestiones a escala humana (Lacey, 1999, p. 128). 
En mis propias palabras, debemos reconocer la importancia de guiarnos por sistemas éticos y sociales que establecen valores y fines, y no por sistemas materiales, ya que estos últimos son ciegos, mientras que los primeros condensan los aspectos propiamente humanos de previsión, dialogo y elección.

\section{LA DISGUSIÓN SOBRE LOS FINES Y LA FINALIDAD DE LA GIENGIA}

En este sentido, la discusión sobre la finalidad de la ciencia debe involucrar la discusión sobre el lugar de la ciencia en la sociedad, sobre los valores involucrados y sobre las implicancias del desarrollo científico y tecnológico. En esta sección, trataré sobre los valores, y en la próxima sobre las implicancias. Pero debe tenerse en cuenta que ambos están asociados a la finalidad de la ciencia, en tanto parte de ella es sin duda tratar de evitar las consecuencias no deseadas en la medida de lo previsible y de lo posible.

$\mathrm{El}$ análisis sobre los valores que deben aplicarse para evaluar el desarrollo científico es diferente según pensemos que la finalidad de la ciencia es principalmente cognitiva o no.

(a) Si pensamos que la finalidad de la ciencia es principalmente cognitiva la discusión sobre valores se liga con el problema del relativismo. Desde el punto de vista académico, esta es principalmente la enseñanza proveniente de la antropología y la sociología (Barnes, 1974; Mulkay, 1979; Cetina \& Mulkay, 1983; Douglas, 1975; Barnes \& Edge, 1982). En mi opinión esta comprensión de la relación ciencia-valor pierde la claridad necesaria para reflexionar sobre la relación ciencia-sociedad. Como lo expresan Schwarz y Thompson, su conclusión principal es que:

la tecnología, la política y las decisiones sociales presentan un entramado ambiguo y, por lo tanto, no podrán nunca ser ajustadas en ningún marco que insista en su carácter definido (Schwarz \& Thompson, 1990, p. 32).

El peligro de esta posición, que no considera posible distinguir lo cognitivo de lo social, es doble. Desde el punto de vista epistemológico, se cae en la relativización del conocimiento científico, en la idea de que las teorías en definitiva responden a los contextos locales de las sociedades que las producen y las mantienen. Tal convicción tiene también consecuencias desde el punto de vista simbólico, dado que se presta a la magnificación del lugar que ocupa el componente retórico en el establecimiento de las teorías aceptadas, y sus prácticas asociadas. De este modo, se corre el riesgo de pensar que la manipulatividad del conocimiento científico es, en definitiva, parte de su propia naturaleza. En este caso, la asociación ciencia-eficacia se convierte en ciencia-mani- 
pulatividad-eficacia. Esta asociación queda muy patente al constituirse en eje de nuevas reconstrucciones de casos centrales de la historia de la ciencia (Cf. Feldhay, 1995; Biagioli,1993).

Este problema no es menor en tanto las propias decisiones de política científica quedan privadas de un concepto claro de ciencia que contribuya al consenso. En mi opinión, el punto señalado por Schwarz y Thompson sólo indica la necesidad de nuevas categorías de análisis para abordar el problema. Creo que también aquí Lacey ofrece una salida viable al centrar el análisis de la ciencia en la distinción de los valores involucrados, en lugar de la búsqueda de criterios o reglas a modo de garantías. Mientras las reglas procuran establecer la objetividad mediante la aplicación de un conjunto finito de pasos formales y - salvo el falsacionismo - teniendo como objetivo central una alta confirmación, por el contrario, la estrategia basada en valores apuesta a defender la imparcialidad de la ciencia en función de la evaluación de los valores cognitivos que esta manifiesta.

Si bien tanto la reflexión como la práctica de la ciencia involucra valores tanto cognitivos como sociales, esto no debe llevarnos a pensar que no podemos distinguir ambos componentes: las teorías se juzgan con valores cognitivos (asociados a la determinación de las teorías y los hechos por ellas explicados), y las prácticas con valores sociales (asociados a la articulación entre políticas científicas y problemas sociales).

(b) Si pensamos que la finalidad de la ciencia no es sólo cognitiva (ciencia como un fin en sí mismo), debemos admitir su finalidad social. Y si bien esto siempre ha sido un supuesto - y quizá por eso mismo - no se ve muchas veces la necesidad de que los mecanismos de diálogo y de superación institucional de las controversias - sobre la finalidad de la ciencia y las implicancias de sus prácticas - deben acompañar y no retrasarse frente al desarrollo de nuevas aplicaciones tecnológicas. Asistimos, en muchos casos, a la existencia y disponibilidad de aplicaciones eficaces, impensables al momento de la última discusión legitimadora sobre la finalidad de esa rama de la tecnología.

\section{REGAPITULANDO: EFIGAGIA Y LEGITIMIDAD}

Volviendo hacia atrás podemos decir que, a diferencia de la eficacia, la legitimidad se logra a través del consenso en marcos institucionales, otorgándole una dimensión humana al desarrollo tecnológico por la referencia a valores, y por la propia elección frente a posibilidades diversas. Por supuesto que, en la medida en que la ciencia, en tanto práctica de actores diferentes, el consenso no se logra de modo necesario. Por este motivo es importante enfatizar el papel de los mecanismos institucionales que, por sobre las diferencias, legitiman el terminar el debate, o dar por finalizado el diálogo. 
En mi opinión, tales procesos de negociación, entre actores que comprenden y valoran de modo diferente, son cruciales y necesarios para la legitimidad de las prácticas científicas a partir del disenso regulado institucionalmente.

Es importante notar aquí que esta discusión es por completo diferente a la explicación del porqué de la eficacia de tales prácticas y de la comprensión de las teorías que las guían. Por este motivo, ha sido un error toda vez que se asoció negociación-retóricarelativismo (independientemente de toda la retórica que pueda haber en tales negociaciones). En todo caso, no es por la retórica que debemos reflexionar sobre el carácter relativo o no del conocimiento científico, sino por la propia posibilidad de comprender de modo diferente en función de diferentes valores cognitivos, o sea, por la existencia de diferentes culturas científicas.

Al mismo tiempo la posibilidad de lograr el consenso estará directamente relacionada con el poder unificador de cada valor sobre un número mayor de los actores involucrados. He señalado al comienzo que las decisiones tomadas en entornos institucionales tienen una dimensión pragmática, donde el tiempo cuenta, y que afectan simultáneamente cuestiones de naturaleza teórica y social.

Al respecto sólo quiero señalar la diferencia relacionada con el impulso y la velocidad de las prácticas científicas en la sociedad. Mientras la necesidad de saldar las inversiones (no sólo económicas, sino también académicas y estratégico-corporativas) y costos de la estructura de producción tecnológica conduce a la necesidad de obtener resultados satisfactorios en el menor tiempo posible, la necesidad de evitar las consecuencias indeseadas imponen un sentido de prudencia que tiende a desacelerar tal desarrollo, ampliando el tiempo de consideración antes de la toma de decisiones.

La distinción entre los dos sentidos de control nos permite también hacer una distinción al reflexionar sobre las inversiones realizadas en ciencia, o en nombre de la ciencia, ya que permite reconocer que, cuando el término "desarrollo" está asociado a la idea de control-1, esto no supone que las cuestiones vinculadas a la idea de control-2 estén saldadas. Y, sin embargo, es sólo por medio de este último camino, o sea, vinculando capacidades tecnológicas con valores sociales, que tales inversiones pueden ser consideradas efectivamente inversiones sociales.

\section{El GONTEXTO DE IMPLicAGión}

Hablar de responsabilidad en la ciencia es hablar de la reflexión sobre sus implicancias, en tanto las acciones responsables también se orientan a partir de proyectar las consecuencias diversas de los cursos posibles de acción. Éstas pueden ser entendidas y valoradas de modo diferente. Los argumentos pueden dirigirse a poner el acento en los 
beneficios o en los riesgos, minimizándolos o maximizándolos, en función de los diversos fines de los actores involucrados.

En mi opinión, la calidad del debate entre tales actores, la posibilidad de consenso y, consiguientemente, la posibilidad de que contribuyan a la cohesión social (en el sentido de la inquietud de Cohen del comienzo y en lugar de hacerlo a la ampliación de las diferencias sociales a la que en muchos casos la tecnología ha contribuido), requieren el mayor acuerdo posible sobre la plataforma conceptual y condiciones para tal diálogo. En lugar de que la controversia se encierre en los criterios y valores de la propia cultura científica de cada bando o individuo, es necesario reconocer la pluralidad de valores involucrados sin que ello sea visto como un obstáculo para alcanzar consensos sobre cuál es el mejor lugar (función) de la ciencia frente a la sociedad.

A continuación quisiera presentar un nuevo contexto socio-epistémico para llevar adelante tal discusión. La idea central es que es necesario debatir de modo simultáneo los aspectos cognitivos, vinculados a la eficacia, y los sociales, vinculados a la legitimidad, y no como dos discusiones casi sin contacto entre sí. Sólo mediante tal simultaneidad en los factores a considerar es posible responder a preguntas del tipo de ¿qué implicancias tienen las prácticas científico-tecnológicas para la sociedad, las generaciones futuras y los ecosistemas?

Más allá de la complejidad obligada que propone este abordaje, no parece haber otro camino para reflexionar sobre la legitimidad y responsabilidad de las prácticas científicas frente a la sociedad. Los contextos habituales de reflexión sobre la ciencia (descubrimiento, justificación y aplicación tecnológica) han conducido, en definitiva, a parcializar una reflexión que debe llevarse adelante de modo conjunto.

Por mi parte sólo pretendo contribuir mediante distinciones conceptuales a la estructuración de este nuevo contexto de reflexión sobre las prácticas científicas; una reflexión participativa que considero no sólo posible, sino necesaria, en la búsqueda de puntos de consenso o equilibrio que conduzcan a prácticas no sólo eficientes, sino también legítimas y responsables.

\subsection{EL VALOR DE GONTROL}

Coincido una vez más con Hugh Lacey en la necesidad de reflexionar sobre si consideramos que el valor de control de la naturaleza debe tender a ser un valor hegemónico. En cualquier caso, creo que una parte significativa de los problemas que encuentra la reflexión sobre las prácticas científicas se origina en una confusión extendida entre dos sentidos diferentes de la idea de "control de la naturaleza": 
- "Control-1" como capacidad tecnológica. En este primer sentido, la idea de control se piensa como un fin en sí mismo, donde el aumento de control es siempre deseable. Podría decirse que esta es la idea técnica de control como valor. Tal capacidad tecnológica puede ser desarrollada sin una finalidad específica. Por otra parte, en los casos donde la finalidad sí está especificada, ésta se refiere a una determinada aplicación o producto tecnológico (Fin-1).

- "Control-2" como dominio de la capacidad tecnológica en función de valores. Este sentido, más amplio, incluye el sentido anterior pero incorpora de modo central la discusión sobre los valores que guían las prácticas científicas. Aquí, la mera referencia a una determinada aplicación o producto tecnológico, no se ve como justificación del uso dado a tal capacidad, sino que se considera necesario que tales resultados representen, además, un avance en el cumplimiento de alguno de los valores consensuados por la sociedad (Fin-2). ${ }^{\mathbf{1}}$

\subsection{Riesgo MATERIAL Y RIESGO SOGIAL}

A este respecto es necesario introducir adicionalmente la distinción entre dos sentidos diferentes de "riesgo".

- Riesgo-1 como riesgo material. Sólo hay responsabilidad en el caso de que algo falle en el diseño tecnológico. En palabras del principal promotor de la nanotecnología, E. Drexler: "nosotros inventamos los mecanismos, ustedes encuentren qué hacer con ellos" (Drexler apud Regis, 1996, p. 47).

- Riesgo-2 como riesgo social. También hay responsabilidad en el caso en que nada falle en el diseño tecnológico, y reconoce que los problemas éticos y sociales vinculados a la ciencia están relacionados con el respeto a los valores mantenidos por la sociedad, y la distribución justa de los beneficios en función del origen de los recursos destinados a su promoción y desarrollo.

\footnotetext{
1 En una conversación personal, ante el planteo de estos dos sentidos del “control”, Lacey me comentó que en su nueva obra él asociaba justamente el primero a la eficacia y el segundo a la legitimidad. En más de un punto estoy de acuerdo con el modo de abordar los problemas por parte de Lacey, y de la necesidad de transitar desde la epistemología criteriológica más extendida, a la epistemología valorativa por él propuesta.
} 


\subsection{Dos ideales de Gonocimiento}

Para poder avanzar también es necesario distinguir entre dos ideales de conocimiento:

- El ideal empirista: máxima testabilidad. De acuerdo a este ideal, las teorías estarán mejor justificadas en la medida en que mejor se haya establecido su concordancia con la evidencia disponible.

- El ideal humanista: máximo desarrollo de nuestras capacidades humanas. De acuerdo a este ideal, la verdad se encuentra por los opuestos - como cuando uno viaja por otras culturas confrontando sus ideas - y la finalidad del conocimiento será el desarrollo de las capacidades intelectuales del hombre, procurando hombres maduros y plenos que interactúen de modo responsable y libre en la sociedad. ${ }^{2}$

\subsection{COMPRENSIÓN AMPLIA Y GOMPRENSIÓN PLENA}

A esta distinción entre dos ideales de conocimiento puede asociársele la distinción de Lacey entre dos modos de comprensión:

- Comprensión amplia: basada en el reconocimiento de objetos y sistemas compatibles con sus principios y leyes - así como de las condiciones particulares que dan cuenta de las diferencias. Suficiente para la eficacia tecnológica y asociada a la idea de control-1.

- Comprensión plena: procura la comprensión del objeto o sistema en sus múltiples aspectos y niveles tanto hacia lo más general como hacia lo más particular. Necesaria para la búsqueda de puntos de acuerdo y equilibrio al tomar decisiones que afecten a la relación ciencia-sociedad.

\subsection{Prácticas eficientes y prácticas Responsables}

De este modo a cada una de las ideas de control les está asociada una idea diferente de fin y, por consiguiente, de responsabilidad. El primer sentido está asociado al ideal empirista de conocimiento, la comprensión amplia y a la eficacia de tales prácticas. El segun-

2 He desarrollado extensamente este punto en Eficacia y letimidad de las prácticas científicas: visitando los textos galileanos con los argumentos de P. Feyerabend, de próxima aparición. 
do sentido está asociado al ideal humanista de conocimiento, la comprensión plena, y a la legitimidad de tales prácticas frente a la sociedad. Ambos responden a motivaciones humanas básicas, sea por buscar la seguridad de la certeza en función del riesgo y la eficacia en un caso, sea por buscar un equilibrio legítimo entre ciencia y sociedad en el otro. Una diferencia fundamental tiene que ver con el corto plazo de lo fines vinculados a muchas aplicaciones tecnológicas y el largo alcance de muchos de sus efectos. Y, al menos en este sentido, las prácticas responsables se vuelven más importantes que las meramente eficaces.

Para ver la relación entre el ideal humanista de conocimiento y las prácticas responsables puede tenerse en cuenta las palabras de W. H. Vanderburg:

Las operaciones tecnológicas tienden a aumentar el control sobre nuestro entorno tanto natural como social con el fin de que produzca lo máximo posible. Es una estrategia que divide y conquista lo individual, lo social y los ecosistemas, para crear un nuevo orden técnico a expensas de la integridad del conjunto. Este nuevo orden global se está convirtiendo en una de las necesidades más complejas en la historia de la humanidad, en el sentido que impone mucho más a la humanidad de lo que la humanidad puede imponerle (Vanderburg, 2000, p. 120).

\section{Conclusión}

Las distinciones realizadas con el fin de bosquejar el contexto de implicación permiten facilitar la identificación de los argumentos expuestos en las controversias científicotecnológicas como persiguiendo un fin u otro, y perteneciendo a planos diferentes de la discusión. Tal identificación es de utilidad en la medida en que permite evitar el encierro al que conduce el verlos como contradictorios, o que sólo puede aceptarse uno debiéndose abandonar al otro.

En mi opinión, este abordaje es fructífero porque permite articular las diferentes culturas cientificas, en su propia identidad, sin buscar tender un manto unificador en la comprensión de los problemas vinculados a la relación ciencia-sociedad. Al mismo tiempo, el contexto de implicación proporcionaría el marco común del que habla B. Lewenstein (2005), que requiere la discusión, dado que el común denominador de las decisiones involucradas afecta a cuestiones de equidad, justicia, poder y libertad. Tal contexto se estructura, entonces, bajo un triple eje ético, político y epistemológico.

El interés de este marco común de discusión consiste en intentar superar el tratamiento parcelado e inconexo de las cuestiones afectadas en contextos epistemológicos diferentes. El contexto de implicación, entonces, puede caracterizar el espacio general 
de la discusión para evaluar tanto los beneficios propuestos por los desarrollos científicos-tecnológicos, como para generar estrategias epistémicas y sociales que permitan puntos de equilibrio y acuerdo, como base de implementación de políticas que tiendan al desarrollo social efectivo.

\author{
Fernando Tula Molina \\ Profesor Adjunto de la Universidad Nacional de Quilmes. \\ Investigador de CONICET, Argentina. \\ ftulamolina@gmail.com
}

\begin{abstract}
This paper propose a new socio-epistemic context to discuss the problems that act upon the interaction between science and society. Using the idea of implication, and the analysis of the social impact of science, I propose some conceptual distinctions that I consider useful to gain consensus in science, technology and society matters. I highlight the pragmatic dimension of decisions involved and the importance of institutional context to legitimate them and affront corresponding responsibilities. The implication context may facilitate taking collective decisions once one understand the need to discuss at the same time the many faces of the problems involved. In substance those faces changes in many ways if we consider matters related to the efficacy or the legitimacy of scientific practices.
\end{abstract}

KeYwords $•$ Philosophy of technology. Implication context. Scientific policy. Epistemology.

\title{
REFERENCIAS BIBLIOGRÁFICAS
}

Barnes, B. Scientific knowledge and sociological theory. London: Routledge and Kegan Paul, 1974.

Barnes, B. \& Edge, D. E. (Ed.). Science in context. Readings in the sociology of science. Cambridge: The MIT Press, 1982.

Biagioli, M. Galileo, courtier: the practice of science in the culture of absolutism. Chicago: Chicago University Press, 1993.

CEtina, K. D. K. \& Mulkay, M. (Ed.). Science observed: newperspectives on the social study of science. London: Sage, 1983.

Cohen, R. S. Social implications of recent technological innovations. In: Durbin, P. T. \& Rapp, F. (Ed.). Philosophy and technology. Dordrecht: Reidel, 1983. p. 35-47.

Douglas, M. Implicit meanings essays in anthropology. London: Routledge and Kegan Paul, 1975.

Durbin, P. T. \& Rapp, F. (Ed.). Philosophy and technology. Dordrecht: Reidel, 1983.

Feldhay, R. Galileo and the church: political inquisition or critical dialogue? Cambridge: Cambridge University Press, 1995 .

LACEY, H. Is science value free? Values and scientific understanding. London/New York: Routledge, 1999.

Lewenstein, B. What counts as a social and ethical issue in nanotechnology? Hyle, International Journal for Philosophy of Chemistry, 11, 1, 2005. p. 5-18. 
Mulkay, M. Science and the sociology of knowledge. Winchester: Allen\& Unwin, 1979.

Regis, E. Nano: the emerging science of nanotechnology. Boston: Back Bay Books, 1996.

Schwarz, M. \& Thompson, M. Divided we stand: re-defining politics, technology, and social choice. Pennsylvania: Penn Press, 1990.

VANDERBURG, W. H. The labyrinth of technology: a preventive technology and economic strategy as a way out. Toronto: University of Toronto Press, 2000.

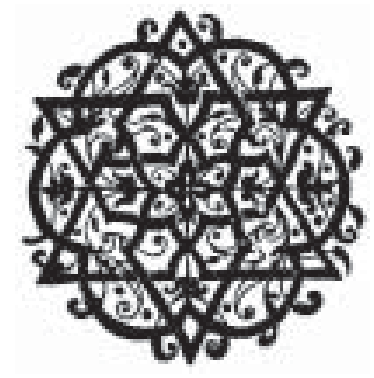

